\title{
Co-jumps and Markov counting systems in random environments
}

\author{
Carles Bretó ${ }^{1}$ \\ Departamento de Estadistica and Instituto Flores de Lemus, Universidad Carlos III de Madrid, C/Madrid 126, Getafe, 28903, Madrid, Spain
}

\begin{abstract}
We provide transition rates for Markov counting systems subject to correlated environmental noises motivated by multi-strain disease models. Such noises induce simultaneous counts, which can help model infinitesimal count correlation (regardless of whether such correlation is due to correlated noises).
\end{abstract}

Keywords: Continuous-time Markov chains, Infinitesimal moments, Compartmental models, Infectious disease models, Environmental stochasticity

\section{Introduction}

Continuous-time stochastic processes have proved to be useful for research in many areas of science. Such processes are naturally defined by infinitesimal parameter functions, like transition rates in the case of Markov chains. Assuming that such parameter functions are subject to external noise has been referred to as a random environment and has proved to be a useful approach to data analysis. A recent example of such applied work is that in Shrestha et al. (2011). This work studied the dynamics governing interactions among multiple infectious pathogens; it found promising results based on Markov counting systems for which multiple transition rates are subject to a single, common external noise. Such common noise results in correlation between these transition rates. Subjecting transition rates to such noise is known to result in a new Markov counting system defined by new rates. However, these new rates have been derived in closed form only for the case of independent noises. This lack of closed-form rates for the case of correlated noises creates uncertainty about the correlated system properties and might make applied researchers reluctant to take advantage of the promising approach presented by Shrestha et al. (2011). To prevent such reticence, this paper considers introducing correlated external noises to the rates of Markov counting systems and provides closed-form expressions for new rates that capture the effect of correlated noise. These new rates are the main contribution of the paper and are based on novel closed-form expressions for the infinitesimal covariances of Markov counting systems. In addition, this unusual focus on system covariances provides an alternative interpretation of correlated noises in terms of simultaneous transitions in non-random environments.

The study of properties of counting processes has benefited among others the fields of epidemiology and ecology, which have relied on Markov counting systems both in deterministic and stochastic environments (the latter sometimes being favoured by empirical evidence). Research in these two disciplines has taken advantage of theoretical investigation of counting systems both historically (Bartlett, 1956; Kermack and McKendrick, 1927) and more recently (Cauchemez and Ferguson, 2008; He et al., 2010). Such counting systems can often be seen as systems of interacting Markov counting processes or Markov counting systems (Bretó and Ionides, 2011), which include networks of queues (Brémaud, 1999) and compartmental models (Jacquez, 1996; Matis and Kiffe, 2000). Markov counting systems are Markov chains and are hence naturally defined by transition rates. Noisy transition rates are often referred to as environmental stochasticity in epidemiology and ecology (Engen et al., 1998). The role of such stochasticity has been extensively studied, including in the context of deterministic ODE skeletons driven by diffusions (Dureau et al., 2013; Hu and Wang, 2011; Ionides et al., 2006; King et al., 2008) and driven by Lévy processes (Bhadra et al., 2011;

\footnotetext{
Email address: carles.breto@uc3m.es (Carles Bretó)

${ }^{1}$ Tel. +34916245855; Fax:+34916249848
} 


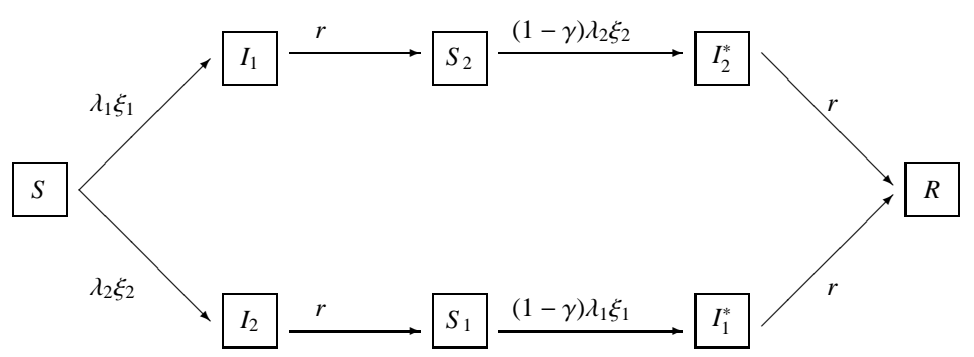

Figure 1: Multi-strain SIR-type compartmental model of Bretó et al. (2009). This model will be used in Section 5 to illustrate our results. Each individual falls in one compartment: $S$, susceptible to both strains ; $I_{1}$, infected with strain $1 ; I_{2}$, infected with strain $2 ; S_{1}$, susceptible to strain 1 (but immune to strain 2); $S_{2}$, susceptible to strain 2 (but immune to strain 1); $I_{1}^{*}$, infected with strain 1 (but immune to strain 2); $I_{2}^{*}$, infected with strain 2 (but immune to strain 1); and $R$, immune to both strains. Regarding demography, births enter $S$ from compartment $B$ (not plotted), at rate $b(t)$ driven by birth data (which is treated as a covariate), and all individuals have a common mortality rate $m$ at which they leave each compartment in the diagram into $D$ (not plotted). Regarding disease dynamics, $r$ is the recovery rate from infection; $\gamma$ measures the strength of cross-immunity between strains; and $\lambda_{i}$ is the per-capita infection rate of strain $i$ with $\xi_{i}$ being the stochastic noise on this rate. Moreover, $\lambda_{i}=\beta(t)\left(I_{i}(t)+I_{i}^{*}(t)\right)^{\alpha} / P(t)+\omega$, where $0 \leq \beta(t)$ is parameterized with a trend and a smooth seasonal component, $0 \leq \omega$ models infections from an environmental reservoir and $0 \leq \alpha \leq 1$ captures inhomogeneous mixing of the population.

Laneri et al., 2010). The role of stochastic environments has also been studied in the context of Markov counting systems, both paying attention to the system probabilistic properties (e.g., Bretó et al., 2009; Marion and Renshaw, 2000; Varughese and Fatti, 2008) and focusing on the biological implications for applications (e.g., Shrestha et al., 2011).

Epidemiological applications have come to consider multiple interacting pathogens and to study them based on counting systems subject to genuinely correlated environmental noise, fitting in the framework provided by Bretó et al. (2009) who formalize the transition rates of the system subject to noise. Pathogen interaction has received attention for some time now (Fenton and Pedersen, 2005; Kamo and Sasaki, 2002), both without considering the role of external noises (Aguiar et al., 2011; Buckee et al., 2011; Reich et al., 2013) and considering it (Bretó et al., 2009; Shrestha et al.,2011). In particular, Shrestha et al. (2011) consider a Markov counting system corresponding to a compartmental model of the susceptible-infectious-recovered type (a simpler version of which is considered by Bretó et al. (2009) and reproduced in Figure 1). In Shrestha et al. (2011), two pathogens co-exist but there are more than two possible different types of infection (depending on the history of past infections of individuals). The rate at which these different types of infection occur are assumed to be subject to a single (common to all infection types) external white noise, making all infection rates correlated. Such rate correlation has been formalized by Bretó et al. (2009), showing that the system subject to noises is a new Markov counting system not only when the noises are independent (in which case they even provide closed-form rates) but also when the noises are correlated, on which this paper focuses. The problem we take up in this paper is providing closed-form transition rates that define Markov counting systems accounting for correlated noises and is made difficult by the lack of closed-form transition probabilities of general systems, working against the inclusion of biologically genuine noise correlation. Closed-form transition probabilities are readily available for basic systems, e.g., those of a Poisson process correspond to a Poisson distribution and those of a linear pure death process to a binomial distribution (Bharucha-Reid, 1960). However, they are not available for general compartmental models, including the system of interacting birth-death processes considered by Shrestha et al. (2011) or that represented in Figure 1 If such closed-form general system transition probabilities were available, then the desired closed-form transition rates might be pursued by direct integration of the noise from those (now randomized) probabilities. Such direct approach is feasible in basic cases (like the bivariate death process of Bretó and Ionides, 2011) but not in more sophisticated models, where the lack of closed-form rates casts a shadow over the appeal of correlated noises in applications of a realistic degree of complexity.

A key downside of lacking closed-form rates is that the promising results of Shrestha et al. (2011) and the genuine biological rationale behind correlated noises may be outweighted by uncertainty about the properties of the model subject to noise and about the interpretation of empirical results, which we seek to prevent with this paper. Shrestha et al. (2011) show that it is feasible to arrive at correct and precise biological conclusions regarding pathogen interaction based on their Markov counting systems with correlated noises. In addition, a heuristic biological justification for correlated noises could be as follows: while localized environmental variations need not affect all types of infection, 
changes at a larger scale in the environment should be expected to, like heat or cold waves. However, unless the properties of the model after subjecting it to correlated noise are clear and appealing, such correlations might be considered a nuisance or something foreign and hence avoided in actual applications, where empirical findings need to be interpreted (which might be done more confidently in the context of simpler models). Providing a tool to help in such interpretation is the ultimate goal of this paper.

The main contribution of this paper is to provide closed-form transition rates for Markov counting systems subject to correlated noises based on the system infinitesimal covariances and to provide an illustration in the context of biological analysis of multi-strain pathogen dynamics. The provided closed-form expressions apply to a broad range of cases considered in the applied literature. They reduce the uncertainty about the model properties by giving a precise definition of the system as formalized in Section 2. In addition, they are motivated by the system infinitesimal covariances derived in Sections 3 and 4 , which allow circumventing the above mentioned problem of unavailable transition probabilities from which to directly integrate out the noise. Our focus on infinitesimal covariances is unusual in the context of Markov counting systems (although as natural as in the context of multivariate diffusions) and leads to the novel closed-form expressions for them provided in Theorem 2 These expressions show that correlated noises induce simultaneous counts and that these in turn induce stronger correlations within the system. Hence, if additional correlation is demanded by data, it could be modelled with random environments. In this case, these environments could be interpreted as devices that generate the needed correlation in a non-random environment, instead of as actual random changes in parameters (very much like parameter randomization can be interpreted as a device to generate over-dispersion). This is illustrated in Section 5, where the rates and interpretation of the role of correlated noises for Figure 1 are given.

\section{Markov counting systems without external noise}

Markov counting systems are defined as Markov chains driven by a collection of interacting counting processes that fully characterizes the transition rates of the system (Bretó et al.,2009) and such definition can often be formalized in a diagram (similar to that in Figure 1). Before formally defining Markov counting systems, we introduce their key aspects. First, consider a population whose members are at any point in time in one (and only one) of $C$ possible stages (or compartments) of their lives, with stages belonging to finite collection $C$. Next, let the number of population members that are at stage $c$ at time $t$ define integer-valued random variables $X_{c}(t)$, which make up the system $\{X(t)\} \equiv$ $\left\{X_{c}(t): c \in C\right\}$. Then, let the number of population members that have transitioned from stage $i$ to stage $j$ by time $t$ define non-decreasing, integer-valued random variables $N_{i j}(t)$, which in turn, for all pairs $(i, j)$ belonging to a collection of allowed transitions $\mathcal{T}$, define the collection of counting processes $\{N(t)\} \equiv\left\{N_{i j}(t):(i, j) \in \mathcal{T}\right\}$. Next, let the collection $\{N(t)\}$ drive the dynamics of the system $\{X(t)\}$ via the "conservation of mass" identity

$$
X_{c}(t)=X_{c}(0)+\sum_{(i, c) \in \mathcal{T}} N_{i c}(t)-\sum_{(c, j) \in \mathcal{T}} N_{c j}(t),
$$

so that changes in $\{X(t)\}$ are the result of changes in $\{N(t)\}$. Mass conservation identity (1) restricts the transitions that can occur in $\{\boldsymbol{X}(t)\}$ as follows. Let $\mathbb{N}_{0}(\mathbb{N})$ be the natural numbers including (excluding) zero and consider initial counts $\boldsymbol{n} \in \mathbb{N}_{0}^{\mathcal{T}}$ and initial system conditions $\boldsymbol{x} \in \mathbb{N}_{0}^{C}$. For any given increments of the collection of counts $\boldsymbol{\ell} \equiv\left\{\ell_{i j}\right.$ : $(i, j) \in \mathcal{T}\} \in \mathbb{N}^{\mathcal{T}}$, the system $\{\boldsymbol{X}(t)\}$ must make transitions $\boldsymbol{u} \equiv\left\{u_{c}: c \in \mathcal{C}\right\} \in \mathbb{Z}^{C}$ with $u_{c}=\sum_{(i, c) \in \mathcal{T}} \ell_{i c}-\sum_{(c, j) \in \mathcal{T}} \ell_{c j}$ Finally, let the following transition rates define the Markov chain $\{\mathbf{X}(t), \mathbf{N}(t)\}$

$$
q(\boldsymbol{x}, \boldsymbol{\ell}) \equiv \lim _{h \downarrow 0} \frac{P(\boldsymbol{N}(t+h)=\boldsymbol{n}+\boldsymbol{\ell}, \boldsymbol{X}(t+h)=\boldsymbol{x}+\boldsymbol{u} \mid \boldsymbol{N}(t)=\boldsymbol{n}, \boldsymbol{X}(t)=\boldsymbol{x})}{h} .
$$

Since the left hand side of (2) only depends on $\boldsymbol{x}$ (and not $\boldsymbol{n}$ ), $\{\boldsymbol{X}(t)\}$ is itself a continuous-time Markov chain and we call it a Markov counting system 2 , which we illustrate with the following example.

\footnotetext{
${ }^{2}$ The transition rates in [2] are time homogeneous, since its left hand side does not depend on $t$. This homogeneity adds clarity to the concepts, results and proofs but can readily be relaxed.
} 
Figure 1 defines a Markov counting system by relying on the concepts of marginal transition rates and of pairwise transition rates, which are necessary for its interpretation and which are also key to study the effect of correlated external noise. Consider the rate at which $k$ population members simultaneously undergo a transition of the $i j$-type (regardless of whether other members undergo other transitions), which can be defined as $q_{i j}(\boldsymbol{x}, k) \equiv \sum_{\boldsymbol{\ell}: \ell_{i j}=k} q(\boldsymbol{x}, \boldsymbol{\ell})$ for $k \in \mathbb{N}$ and which we call the $(i, j)$ marginal transition rate. Marginal transition rates of size one $q_{i j}(\boldsymbol{x}, 1)$ are the labels on the arrows in Figure 1. Marginal rates of sizes greater than one do not appear in Figure 1 because they are assumed to be zero. Another assumption needed to interpret Figure 1 is that there are no co-jumps of different types. To formalize this second assumption, consider the rate at which $\boldsymbol{k}=\left(k_{i j}, k_{i^{\prime} j^{\prime}}\right)$ population members simultaneously undergo transitions of the $(i, j)$ and $\left(i^{\prime}, j^{\prime}\right)$ types (regardless of whether other members undergo other transitions), which can be defined as $q_{i j, i^{\prime} j^{\prime}}(\boldsymbol{x}, \boldsymbol{k}) \equiv \sum_{\ell: \ell_{i j}=k_{i j}, \ell_{i^{\prime} j^{\prime}}=k_{i^{\prime} j^{\prime}}} q(\boldsymbol{x}, \boldsymbol{\ell})$ for $\boldsymbol{k} \in\left\{\mathbb{N}_{0}^{2}-(0,0)\right\}$ and which we call the $(i, j)-\left(i^{\prime}, j^{\prime}\right)$ pairwise transition rate. Requiring all pairwise transition rates to satisfy $q_{i j, i^{\prime} j^{\prime}}(\boldsymbol{x},(1,0))=q_{i j}(\boldsymbol{x}, 1)$ and $q_{i j, i^{\prime} j^{\prime}}(\boldsymbol{x},(0,1))=q_{i^{\prime} i^{\prime}}(\boldsymbol{x}, 1)$ guarantees no co-jumps and allows interpreting figures such as Figure 1 as Markov chains (see Anderson and Mav, 1991; Brémaud, 1999; Jacquez, 1996). Such interpretation also assumes that all non-zero rates $q_{i j}(\boldsymbol{x}, 1)$ are deterministic functions of the chain state $\boldsymbol{x}$ and not subject to external noise.

\section{Correlated external noise in bivariate death Markov counting systems}

Introducing correlated noise to the rates is easier if one considers two independent death processes and results in both co-jumps and in infinitesimal covariance as stated in Proposition 1 below, which considers a common multiplicative gamma external noise, a common death rate, and which will later be useful when considering general Markov counting systems. Proposition 1 was proved in Bretó and Ionides (2011) and we state it here to make the paper selfcontained. We state it in terms of our notation for Markov counting systems, after introducing our notation for the noise. The noise affecting the individual death rates is assumed to be continuous-time white noise obtained from a gamma process, which is also the choice of noise in Bretó et al. (2009) and Shrestha et al. (2011). Gamma white noise is defined as $\{\xi(t)\} \equiv\{d \Gamma(t) / d t\}$ with $\Gamma(t) \sim \operatorname{Gamma}(t / \tau, \tau), E[\Gamma(t)]=t$, and $V[\Gamma(t)]=\tau t$, so that $\tau$ parameterizes the magnitude of the noise.

Proposition 1 (Proposition 7 of Bretó and Ionides, 2011). Consider the bivariate Markov counting system $\{\boldsymbol{Y}(t)\} \equiv$ $\left\{\left(Y_{1}(t), Y_{2}(t)\right)\right\}$ defined by counting processes $\left\{\left(N_{Y_{1} D}(t), N_{Y_{2} D}(t)\right)\right\}$ through mass conservation equations

$$
Y_{i}(t)=Y_{i}(0)-N_{Y_{i} D}(t),
$$

and by transition rates $q_{Y_{i} D}\left(y_{i}, 1\right)=\delta y_{i} \mathbb{I}\left\{0<y_{i}\right\}$ i.e., two independent linear death processes having equal individual death rate $\delta \in R^{+}$and initial population sizes $Y_{i}(0)$. Consider subjecting both $q_{Y_{i} D}\left(y_{i}, 1\right)$ to a common gamma white noise, which defines counting processes $\left\{N_{\tilde{Y}_{i} \tilde{D}}(t)\right\}=\left\{N_{Y_{i} D}(\Gamma(t))\right\}$ and the corresponding Markov counting system $\{\tilde{\boldsymbol{Y}}(t)\}$, i.e., two death processes each having stochastic rate $\delta \xi(t)$. Then, the transition rates of $\{\tilde{\boldsymbol{Y}}(t)\}$ correspond to pairwise transition rates, letting $k_{i}=k_{\tilde{Y}_{i} \tilde{D}} \in\left\{\mathbb{N}_{0}^{2}-(0,0): k_{i} \leq \tilde{y}_{i}\right\}$,

$$
q_{\tilde{Y}_{1} \tilde{D}, \tilde{Y}_{2} \tilde{D}}\left(\left(\tilde{y}_{1}, \tilde{y}_{2}\right),\left(k_{1}, k_{2}\right)\right)=\left(\begin{array}{l}
\tilde{y}_{1} \\
k_{1}
\end{array}\right)\left(\begin{array}{l}
\tilde{y}_{2} \\
k_{2}
\end{array}\right) \sum_{j=0}^{k_{1}+k_{2}}\left(\begin{array}{c}
k_{1}+k_{2} \\
j
\end{array}\right)(-1)^{k_{1}+k_{2}-j+1} \tau^{-1} \ln \left(1+\delta \tau\left(\tilde{y}_{1}+\tilde{y}_{2}-j\right)\right) \text {. }
$$

Furthermore, the infinitesimal covariance between $\left\{N_{\tilde{Y}_{1} \tilde{D}}(t)\right\}$ and $\left\{N_{\tilde{Y}_{2} \tilde{D}}(t)\right\}$ is

$$
\lim _{h \downarrow 0} h^{-1} \operatorname{Cov}\left[N_{\tilde{Y}_{1} \tilde{D}}(t+h)-N_{\tilde{Y}_{1} \tilde{D}}(t), N_{\tilde{Y}_{2} \tilde{D}}(t+h)-N_{\tilde{Y}_{2} \tilde{D}}(t) \mid \tilde{\boldsymbol{Y}}(t)=\tilde{\boldsymbol{y}}\right]=\tilde{y}_{1} \tilde{y}_{2} \tau^{-1} \ln \left(\frac{(1+\delta \tau)^{2}}{1+2 \delta \tau}\right)>0 .
$$

The common rate assumption of Proposition 1 can be relaxed at the cost of more complex closed-form expressions for the covariance and for the pairwise rates. Although different death rates are assumed in the interacting death processes of Figure 1 they will be assumed to be equal for the sake of simplicity when we illustrate in Section 5 our results for general Markov counting systems (which hold regardless of whether individual death rates are equal). 


\section{Correlated external noise in general Markov counting systems}

Consider generalizing Proposition 1 to general Markov counting systems, which will lead us to defining infinitesimal covariances of such general systems. Consider a general Markov counting system as defined by transition rates (2) that satisfies the standard assumptions to interpret Figure 1 of neither multiple jumps nor co-jumps and denote such system by $\{\boldsymbol{W}(t)\}$. Consider now subjecting some (or all) transition rates of $\{\boldsymbol{W}(t)\}$ to a collection of (possibly correlated, not necessarily gamma) white noises derived from $\{\boldsymbol{\Gamma}(t)\}$ (analogously to Section 3) and call the resulting process $\{\tilde{\boldsymbol{W}}(t)\}$. The transitions rates of $\{\tilde{\boldsymbol{W}}(t)\}$ could be obtained by integrating out the noises $\{\boldsymbol{\Gamma}(t)\}$ from the (now randomized) transition probabilities appearing inside the limit in (2) as follows. Consider the collection of randomized time increments $\boldsymbol{H} \equiv\left\{H_{i j}:(i, j) \in \mathcal{T}\right\}$, necessarily with $E\left[H_{i j}\right]=h$. The nature of $H_{i j}$ depends on whether $q_{i j}(\boldsymbol{w}, 1)$ is subject to noise: if yes, then $H_{i j}$ is the corresponding noise random variable with density $f_{H_{i j}}$; if not, then it is the degenerate random variable $H_{i j}=h$. Then, provided they exist, the transition rates of $\{\tilde{\boldsymbol{W}}(t)\}$ are

$$
q(\tilde{\boldsymbol{w}}, \boldsymbol{\ell})=\lim _{h \downarrow 0} \int \frac{P(\boldsymbol{N}(t+\boldsymbol{s})=\boldsymbol{n}+\boldsymbol{\ell}, \boldsymbol{W}(t+\boldsymbol{s})=\boldsymbol{w}+\boldsymbol{u} \mid \boldsymbol{N}(t)=\boldsymbol{n}, \boldsymbol{W}(t)=\boldsymbol{w})}{\boldsymbol{s}} f_{\boldsymbol{H}}(\boldsymbol{s}) d \boldsymbol{s} .
$$

While such direct integration of the noise in equation (3) was straightforward for the bivariate death process of Proposition 1, it is not so straightforward for more sophisticated models, like the one in Figure 10 (Bretó et al., 2009) or similar models (Shrestha et al., 2011). Hence, instead of obtaining the new transition rates by direct integration, we propose constructing such new rates by directly specifying transition rates that produce the same infinitesimal covariance as that produced by introducing noise to appropriate bivariate systems. In the case of Figure 1, noises $\xi_{i}(t)$ affect the rate of death processes $\left\{N_{S I_{i}}\right\}$ and $\left\{N_{S_{i} I_{i}^{*}}\right\}$. In this case, our proposal amounts to specifying a new set of transition rates for the system represented by Figure 1 such that the infinitesimal covariance between $\left\{N_{S I_{i}}\right\}$ and $\left\{N_{S_{i} I_{i}^{*}}\right\}$ matches the covariance given by Proposition 1 between $\left\{N_{Y_{1} D}\right\}$ and $\left\{N_{Y_{2} D}\right\}$. To do this, we first derive closed-form expressions for the infinitesimal covariances between two counting processes involved in a Markov counting system.

\subsection{Infinitesimal covariance of Markov counting systems}

Define the infinitesimal covariances of a general Markov counting system $\{\boldsymbol{X}(t)\}$ as defined in Section 2 as the collection

$$
\left\{\boldsymbol{\sigma}_{d \boldsymbol{X}}(\boldsymbol{x})\right\} \equiv\left\{\sigma_{d \boldsymbol{X}}^{i j, i^{\prime} j^{\prime}}(\boldsymbol{x}):(i, j) \neq\left(i^{\prime}, j^{\prime}\right) \in \mathcal{T}\right\}
$$

of infinitesimal covariances between counting processes $\left\{N_{i j}(t)\right\}$ and $\left\{N_{i^{\prime} j^{\prime}}(t)\right\}$ :

$$
\sigma_{d \boldsymbol{X}}^{i j, i^{\prime} j^{\prime}}(\boldsymbol{x}) \equiv \lim _{h \downarrow 0} h^{-1} \operatorname{Cov}\left[N_{i j}(t+h)-N_{i j}(t), N_{i^{\prime} j^{\prime}}(t+h)-N_{i^{\prime} j^{\prime}}(t) \mid \boldsymbol{X}(t)=\boldsymbol{x}\right] .
$$

Our closed-form expressions below require one moment existence condition. Similar conditions were required by Theorem 1 of Bretó and Ionides (2011) to provide closed-form expressions for the infinitesimal mean and variance of Markov counting processes. Since Bretó and Ionides (2011) call their condition for the mean $\left(P 1^{\star}\right)$ and that for the variance $\left(P 2^{\star}\right)$, we shall call our condition for covariances $\left(P 3^{\star}\right) .\left(P 3^{\star}\right)$ is related to the number of transitions occurring in the Markov counting system over a time interval. This number of transitions is related not only to the sizes of the increments of each counting process $\left\{N_{i j}(t)\right\}$ but also to the overall rate at which these increments occur, which we call the rate function of the Markov counting system and define as

$$
\lambda(\boldsymbol{x}) \equiv \lim _{h \downarrow 0} \frac{1-P\left(N_{i j}(t+h)-N_{i j}(t)=0 \text { for all }(i, j) \in \mathcal{T} \mid \boldsymbol{X}(t)=\boldsymbol{x}\right)}{h} .
$$

This quantity is also know as the intensity of the process in the point process literature (Daley and Vere-Jones, 2003). If the rate function satisfies that $\lambda(\boldsymbol{x})=\sum_{\ell} q(\boldsymbol{x}, \boldsymbol{\ell})<\infty$ for all $\boldsymbol{x}$, then the process is said to be stable and conservative.

Consider stochastically bounding the rate function and the increment of each pair of counting processes over $[t, t+\bar{h}]$ by:

$$
\bar{\Lambda}(t) \equiv \sup _{t \leq s \leq t+\bar{h}} \lambda(\boldsymbol{X}(s)), \quad \bar{Z}_{i j, i^{\prime} j^{\prime}}(t) \equiv \sup \left\{\sup _{t \leq s \leq t+\bar{h}} d N_{i j}(s), \sup _{t \leq s \leq t+\bar{h}} d N_{i^{\prime} j^{\prime}}(s)\right\} .
$$


A combination of these two bounds gives the following property:

$\mathbf{P 3}^{\star}$. For each $t, \boldsymbol{x}$ and $(i, j) \neq\left(i^{\prime}, j^{\prime}\right)$ there is some $\bar{h}>0$ such that $E\left[\bar{Z}_{i j, i^{\prime}, j^{\prime}}^{2}(t) \bar{\Lambda}(t) \mid \boldsymbol{X}(t)=\boldsymbol{x}\right]<\infty$.

Property $\left(P 3^{\star}\right)$ requires that the Markov counting system does not have an explosive behaviour and holds, for example, for SIR-type compartmental models like that of Figure 1] as shown in Section 5. It suffices to guarantee that infinitesimal covariances exist and that are given by the expression in Theorem 2 below.

Theorem 2 (Infinitesimal covariances of a Markov counting system). Let $\{X(t)\}$ be a time homogeneous Markov counting system defined by counting processes $\{\boldsymbol{N}(t)\}$ and by transition rates $q(\boldsymbol{x}, \boldsymbol{\ell})$ as in (2) that is stable and conservative. Supposing $\left(P 3^{\star}\right)$, the infinitesimal covariance between $\left\{N_{i j}(t)\right\}$ and $\left\{N_{i^{\prime} j^{\prime}}(t)\right\}$ is

$$
\sigma_{d \boldsymbol{X}}^{i j, i^{\prime} j^{\prime}}(\boldsymbol{x})=\sum_{\boldsymbol{k}} k_{i j} k_{i^{\prime} j^{\prime}} q_{i j, i^{\prime} j^{\prime}}(\boldsymbol{x}, \boldsymbol{k})
$$

Theorem 2 generalizes Theorem 1 of Bretó and Ionides (2011) to covariances and is proved in Appendix A.

\section{Transition rates of SIR-type models subject to external correlated noises}

Theorem 2 can be used to show that, after minimal simplifications to add clarity to our contribution, the system represented in Figure 1 can be defined by transition rates that reproduce the effects (identified in Proposition 1 of correlated noises. First, consider the Markov chain $\{\boldsymbol{Z}(t)\} \equiv\left\{\left(S(t), I_{1}(t), I_{2}(t), S_{1}(t), S_{2}(t), I_{1}^{*}(t), I_{2}^{*}(t), R(t)\right)\right\}$ specified by Figure 1. The standard interpretation of Figure 1 gives the transition rates for $\{\boldsymbol{Z}(t)\}$ in Table 1 (e.g., letting the $\xi_{i}$ be deterministic constants). Next, before considering adding noise to $\{\boldsymbol{Z}(t)\}$, we make the following simplifications to $\{\boldsymbol{Z}(t)\}$ so that the contributions in this paper can be presented more clearly: (i) instead of a time-inhomogeneous birth rate $b(t)$, births compensate deaths so that the total population size remains constant and is equal to $P<\infty$, as in Shrestha et al. (2011); (ii) instead of a time-inhomogeneous infection rate $\beta(t)$ within $\lambda_{i}$, this rate is constant and equal to $\beta$; and (iii) instead of different transition rates from $S$ to $I_{i}$ and from $S_{i}$ to $I_{i}^{*}$, these rates are both $\lambda_{i} \xi_{i}(t)$, i.e., $\gamma=0$. Simplifications (i) and (ii) impose time-homogeneity, which allows for a simpler notation in the rest of the paper. Simplification (iii) allows for simpler transition rates and covariance closed-form expressions. Now, let stable, conservative Markov chain $\{\tilde{\boldsymbol{Z}}(t)\}$ be defined by the rates of Table 1 modified according to (i)-(iii) and by non-zero pairwise rates of transitions involving the $\xi_{i}$ equal to those in Proposition 1 as follows:

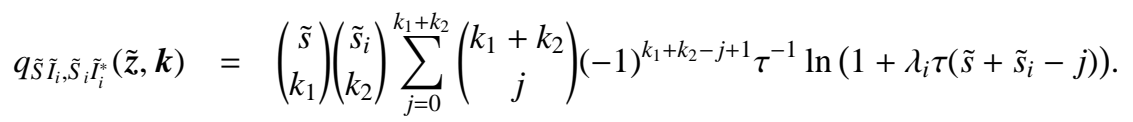

System $\{\tilde{\boldsymbol{Z}}(t)\}$ defined by these rates satisfies $\left(P 3^{\star}\right)$, since letting the fixed population size be $\tilde{P}$,

$$
\begin{aligned}
\lambda(\tilde{\boldsymbol{Z}}(t)) & =m(\tilde{P}-\tilde{S}(t))+r\left(\tilde{I}_{1}(t)+\tilde{I}_{2}(t)+\tilde{I}_{1}^{*}(t)+\tilde{I}_{2}^{*}(t)\right)+\sum_{\boldsymbol{k}} q_{\tilde{S}_{I_{1}, \tilde{S}_{1} \tilde{I}_{1}^{*}}}(\tilde{\boldsymbol{Z}}(t), \boldsymbol{k})+\sum_{\boldsymbol{k}} q_{\tilde{S} \tilde{I}_{2}, \tilde{S}_{2} \tilde{I}_{2}^{*}}(\tilde{\boldsymbol{Z}}(t), \boldsymbol{k}) \\
& \leq\left(m+r+\lambda_{1}+\lambda_{2}\right) \tilde{P}
\end{aligned}
$$

where the inequality follows by substituting all compartments by $\tilde{P}$ and because

$$
\sum_{\boldsymbol{k}} q_{\tilde{S} \tilde{I}_{i}, \tilde{S}_{i} \tilde{I}_{i}^{*}}(\tilde{\mathbf{Z}}(t), \boldsymbol{k})=\tau^{-1} \ln \left(1+\tau \lambda_{i}\left(\tilde{S}(t)+\tilde{S}_{i}\right)\right) \leq \lambda_{i} \tilde{P}
$$

(as follows from the properties of the binomial gamma process of Bretó and Ionides, 2011). Since (7) is not timevarying, it also bounds $\bar{\Lambda}(t)$ involved in $\left(P 3^{\star}\right)$. Similarly, $\tilde{P}$ is an upper bound for the increments: $\bar{Z}_{i j, i^{\prime} j^{\prime}}(t) \leq \tilde{P}$, so that

$$
E\left[\bar{Z}_{i j, i^{\prime} j^{\prime}}^{2}(t) \bar{\Lambda}(t) \mid \tilde{Z}(t)=\tilde{z}\right] \leq\left(m+r+\lambda_{1}+\lambda_{2}\right) \tilde{P}^{3}
$$


$\left(P 3^{\star}\right)$ can be analogously verified for the bivariate system $\{\tilde{\boldsymbol{Y}}(t)\}$ of Proposition 1 by assuming, for example, that the initial population sizes are deterministic, i.e., for fixed $y_{i}(0)=\tilde{y}_{i}(0)$

$$
\lambda(\tilde{\boldsymbol{Y}}(t))=\sum_{\boldsymbol{k}} q_{\tilde{Y}_{1} \tilde{D}, \tilde{Y}_{2} \tilde{D}}(\tilde{\boldsymbol{Y}}(t), \boldsymbol{k})=\tau^{-1} \ln \left(1+\tau \delta\left(\tilde{Y}_{1}(t)+\tilde{Y}_{2}(t)\right)\right) \leq \delta\left(\tilde{y}_{1}(0)+\tilde{y}_{2}(0)\right) .
$$

Hence, it follows directly from Theorem 2 that

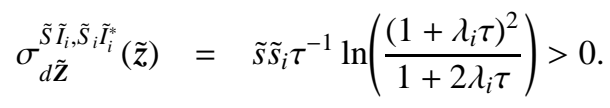

Equation (8) can be interpreted as follows. First, the effects of introducing correlated noises in the bivariate system of Proposition 1 can be reproduced in more general systems. Second, it permits an alternative interpretation of correlated noises $\xi_{i}(t)$ in a non-random environment context. These noises have effectively been integrated out in $\{\tilde{\boldsymbol{Z}}(t)\}$, which can be seen as a regular Markov chain in a non-random environment, with the caveat that it now allows for simultaneous co-transitions that drive the new infinitesimal correlations.

Table 1: Transition rates according the standard interpretation of Figure 1 as a continuous-time Markov chain with rate function $\lambda_{Z}(z) \equiv$ $\sum_{(i, j) \in \mathcal{T}} q_{i j}(z, 1)$ and with all marginal rates $q_{i j}(z, k)$ for $k>1$ and all pairwise transition rates $q_{i j, i^{\prime} j^{\prime}}(z, k)$ assumed to be zero.

\begin{tabular}{c|cccccccccc}
\hline \hline$N_{i j}$ & $N_{S I_{i}}$ & $N_{I_{i} S_{i}}$ & $N_{S_{i} I_{i}^{*}}$ & $N_{I_{i}^{*} R}$ & $N_{B S}$ & $N_{S D}$ & $N_{I_{i} D}$ & $N_{S_{i} D}$ & $N_{I_{i}^{*} D}$ & $N_{R D}$ \\
\hline$q_{i j}(z, 1)$ & $\lambda_{i} \xi_{i}$ & $r$ & $(1-\gamma) \lambda_{i} \xi_{i}$ & $r$ & $b(t)$ & $m$ & $m$ & $m$ & $m$ & $m$ \\
\hline
\end{tabular}

\section{Acknowledgements}

This work was supported by Spanish Government Project ECO2012-32401 and Spanish Program Juan de la Cierva (JCI-2010-06898).

\section{References}

Aguiar, M., Stollenwerk, N., Kooi, B. W., 2011. The Stochastic Multi-strain Dengue Model: Analysis of the Dynamics. In: Simos, T. E., Psihoyios, G., Tsitouras, C., Anastassi, Z. (Eds.), American Institute of Physics Conference Series. Vol. 1389 of American Institute of Physics Conference Series. pp. 1224-1227.

Anderson, R. M., May, R. M., 1991. Infectious Diseases of Humans. Oxford University Press, Oxford.

Bartlett, M. S., 1956. Deterministic and stochastic models for recurrent epidemics. In: Proc. of the Third Berkeley Symposium on Mathematical Statistics and Probability. Univ. California Press, Berkeley, pp. 81-109.

Bhadra, A., Ionides, E. L., Laneri, K., Pascual, M., Bouma, M., Dhiman, R. C., 2011. Malaria in Northwest India: Data analysis via partially observed stochastic differential equation models driven by Lévy noise. Journal of the American Statistical Association 106, 440-451.

Bharucha-Reid, A. T., 1960. Elements of the Theory of Markov Processes and their Applications. McGraw-Hill.

Brémaud, P., 1999. Markov Chains: Gibbs Fields, Monte Carlo Simulation, and Queues. Springer, New York.

Bretó, C., 2012. On infinitesimal dispersion of multivariate Markov counting systems. Statistics and Probability Letters 82, 720-725.

Bretó, C., He, D., Ionides, E., King, A., 2009. Time series analysis via mechanistic models. Annals of Applied Statistics 3, 319-348.

Bretó, C., Ionides, E., 2011. Compound Markov counting processes and their applications to modeling infinitesimally over-dispersed systems. Stochastic Processes and their Applications 121, 2571-2591.

Buckee, C. O., Recker, M., Watkins, E. R., Gupta, S., 2011. Role of stochastic processes in maintaining discrete strain structure in antigenically diverse pathogen populations. Proceedings of the National Academy of Sciences 108 (37), 15504-15509.

Cauchemez, S., Ferguson, N. M., 2008. Likelihood-based estimation of continuous-time epidemic models from time-series data: application to measles transmission in London. Journal of The Royal Society Interface 5, 25, 885-897.

Cox, D., Isham, V., 1980. Point Processes. Chapman \& Hall.

Daley, D., Vere-Jones, D., 2003. An Introduction to the Theory of Point Processes. Volume I: Elementary Theory and Methods. Springer.

Dureau, J., Kalogeropoulos, K., Baguelin, M., 2013. Capturing the time-varying drivers of an epidemic using stochastic dynamical systems. Biostatistics 14 (3), 541-555.

Engen, S., Bakke, O., Islam, A., 1998. Demographic and environmental stochasticity: Concepts and definitions. Biometrics 54, 840-846.

Fenton, A., Pedersen, A., 2005. Community epidemiology framework for classifying disease threats. Emerging Infectious Diseases 11, $1815-1821$.

He, D., Ionides, E., King, A., 2010. Plug-and-play inference for disease dynamics: Measles in large and small populations as a case study. Journal of the Royal Society Interface 7, 271-283. 
Hu, G., Wang, K., 2011. Stability in distribution of competitive lotkavolterra system with markovian switching. Applied Mathematical Modelling 35 (7), 3189-3200.

Ionides, E. L., Bretó, C., King, A. A., 2006. Inference for nonlinear dynamical systems. Proceedings of the National Academy of Sciences of the USA $103,18438-18443$.

Jacquez, J. A., 1996. Compartmental Analysis in Biology and Medicine. 3rd edition, BioMedware, Ann Arbor, MI.

Kamo, M., Sasaki, A., 2002. The effect of cross-immunity and seasonal forcing in a multi-strain epidemic model. Physica D 165, $228-241$.

Kermack, W. O., McKendrick, A. G., 1927. A contribution to the mathematical theory of epidemics. Proceedings of the Royal Society of London, Ser. A $115,700-721$.

King, A., Ionides, E., Pacual, M., Bouna, M., 2008. Inapparent infections and cholera dynamics. Nature 454, 877-880.

Laneri, K., Bhadra, A., Ionides, E., Bouma, M., Dhiman, R., Yadav, R. S., Pascual, M., 2010. Forcing versus feedback: Epidemic malaria and monsoon rains in Northwest India. PLoS Computational Biology 6.

Marion, G., Renshaw, E., 2000. Stochastic modelling of environmental variation for biological populations. Theoretical Population Biology 57, 197-217.

Matis, J. H., Kiffe, T. R., 2000. Stochastic Population Models. A Compartmental Perpective. Springer.

Reich, N. G., Shrestha, S., King, A. A., Rohani, P., Lessler, J., Kalayanarooj, S., Yoon, I.-K., Gibbons, R. V., Burke, D. S., Cummings, D. A. T., 2013. Interactions between serotypes of dengue highlight epidemiological impact of cross-immunity. Journal of The Royal Society Interface $10(86)$

Ross, S., 1996. Stochastic Processes, 2nd Edition. Wiley.

Shrestha, S., King, A., Rohani, P., 2011. Statistical inference for multi-pathogen systems. PLoS Computational Biology 7(8): e1002135.

Varughese, M., Fatti, L., 2008. Incorporating environmental stochasticity within a biological population model. Theoretical Population Biology 74, $115-129$.

\section{Appendix A. Proof of Theorem 2}

Proof. First, we prove that the infinitesimal covariance equals the infinitesimal cross-product under condition $\left(P 3^{\star}\right)$. Let $\Delta N_{i j}(t) \equiv N_{i j}(t+h)-N_{i j}(t)$ and analogously for all other subindices and counting processes in this proof. Since $\left(P 3^{\star}\right)$ implies $\left(P 1^{\star}\right)$ in Theorem 1 of Bretó (2012), it follows that $E\left[\Delta N_{i j}(t) \mid \boldsymbol{X}(t)=\boldsymbol{x}\right]=h \sum_{k} k q_{i j}(\boldsymbol{x}, k)+o(h)$. Then

$$
\begin{aligned}
\lim _{h \downarrow 0} h^{-1} \operatorname{Cov}\left[\Delta N_{i j}(t), \Delta N_{i^{\prime} j^{\prime}}(t) \mid \boldsymbol{X}(t)=\boldsymbol{x}\right] & =\lim _{h \downarrow 0} h^{-1}\left\{E\left[\Delta N_{i j}(t) \Delta N_{i^{\prime} j^{\prime}}(t) \mid \boldsymbol{X}(t)=\boldsymbol{x}\right]-o(h)\right\} \\
& =\lim _{h \downarrow 0} h^{-1} E\left[\Delta N_{i j}(t) \Delta N_{i^{\prime} j^{\prime}}(t) \mid \boldsymbol{X}(t)=\boldsymbol{x}\right] .
\end{aligned}
$$

The rest of this proof follows closely the proof of Theorem 1 in Bretó (2012). While that theorem provided expressions for the infinitesimal mean and variance of $\{\boldsymbol{X}(t)\}$, this one provides them for the covariances.

All probabilities and expectations in this proof are conditional on $\boldsymbol{X}(t)=\boldsymbol{x}$ (in addition to other conditioning, where appropriate). Define the following: (i) let $\left\{\bar{N}_{i j}(t)\right\}$ be a process such that, conditional on $\bar{\Lambda}(t)$ and $\bar{Z}(t) \equiv \bar{Z}_{i j, i^{\prime} j^{\prime}}(t)$, realizations of $\left\{\bar{N}_{i j}(t)\right\}$ are those of a compound Poisson process (Cox and Isham, 1980) with Poisson event rate $\bar{\Lambda}(t)$ and degenerate jump or batch size distribution (Daley and Vere-Jones, 2003) with mass one at $\bar{Z}(t)$, i.e., a process with jumps arriving according to the Poisson process and for which the size of the jumps is $\bar{Z}(t)$; and (ii) let $S$ be the event that there is exactly one transition time occurring in the interval $[t, t+h]$ in the Markov counting system $\{\boldsymbol{X}(t)\}$. Then,

$$
E\left[\Delta N_{i j}(t) \Delta N_{i^{\prime} j^{\prime}}(t)\right]=E\left[\Delta N_{i j}(t) \Delta N_{i^{\prime} j^{\prime}}(t) \mathbb{I}\{S\}\right]+E\left[\Delta N_{i j}(t) \Delta N_{i^{\prime} j^{\prime}}(t) \mathbb{I}\left\{S^{c}\right\}\right] .
$$

Consider the first term on the right hand side of (A.1). Let $S_{i j, i^{\prime} j^{\prime}} \subset S$ be the event that there is exactly one transition time occurring in the interval $[t, t+h]$ in the MCS $\{\boldsymbol{X}(t)\}$ and that this transition increases both the $\left\{N_{i j}(t)\right\}$ and the

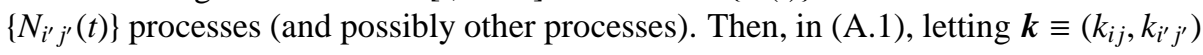

$$
\begin{aligned}
E\left[\Delta N_{i j}(t) \Delta N_{i^{\prime} j^{\prime}}(t) \mathbb{I}\{S\}\right] & =E\left[\Delta N_{i j}(t) \Delta N_{i^{\prime} j^{\prime}}(t) \mid S_{i j, i^{\prime} j^{\prime}}\right] \times P\left(S_{i j, i^{\prime} j^{\prime}} \mid S\right) \times P(S) \\
& =\sum_{\boldsymbol{k}} k_{i j} k_{i{ }^{\prime} j^{\prime}} \frac{q_{i j, i^{\prime} j^{\prime}}(\boldsymbol{x}, \boldsymbol{k})}{\sum_{\boldsymbol{k}} q_{i j, i^{\prime} j^{\prime}}(\boldsymbol{x}, \boldsymbol{k})} \times \frac{\sum_{\boldsymbol{k}} q_{i j, i^{\prime} j^{\prime}}(\boldsymbol{x}, \boldsymbol{k})}{\lambda(\boldsymbol{x})} \times[h \lambda(\boldsymbol{x})+o(h)] \\
& =h \sum_{\boldsymbol{k}} k_{i j} k_{i^{\prime} j^{\prime}} q_{i j, i^{\prime} j^{\prime}}(\boldsymbol{x}, \boldsymbol{k})+o(h)
\end{aligned}
$$


where $P(S)$ in A.2) follows by a standard result on Markov chains (see for example: Ross, 1996, page 492).

To finish the proof, we show that the second term on the right hand side of A.1 disappears infinitesimally. Let $\bar{S}$ be the event that there is exactly one transition time occurring in the interval $[t, t+h]$ in the compound Poisson process $\left\{\bar{N}_{i j}(t)\right\}$. Since the random variable $\Delta N_{i j}(t) \Delta N_{i^{\prime} j^{\prime}}(t)$ is stochastically smaller than $\left(\Delta \bar{N}_{i j}(t)\right)^{2}$,

$$
\begin{aligned}
E\left[\Delta N_{i j}(t) \Delta N_{i^{\prime} j^{\prime}}(t) \mathbb{I}\left\{S^{c}\right\}\right] & \leq E\left[\left(\Delta \bar{N}_{i j}(t)\right)^{2} \mathbb{I}\left\{\bar{S}^{c}\right\}\right] \\
& =E\left[E\left[\left(\Delta \bar{N}_{i j}(t)\right)^{2} \mid \bar{\Lambda}(t), \bar{Z}(t)\right]\right]-E\left[E\left[\left(\Delta \bar{N}_{i j}(t)\right)^{2} \mathbb{I}\{\bar{S}\} \mid \bar{\Lambda}(t), \bar{Z}(t)\right]\right] \\
& =(E\left[\bar{Z}^{2}(t) \bar{\Lambda}(t) h\right]+\underbrace{E\left[\bar{Z}^{2}(t) \bar{\Lambda}^{2}(t)\right] h^{2}}_{=o(h)})-E\left[\bar{Z}^{2}(t) \bar{\Lambda}(t) h \exp \{-h \bar{\Lambda}(t)\}\right] \\
& =E\left[\bar{Z}^{2}(t) \bar{\Lambda}(t) h(1-\exp \{-h \bar{\Lambda}(t)\})\right]+o(h)
\end{aligned}
$$

where A.4 follows as in A.1 , and (A.5) follows by the properties of the compound Poisson distribution. Since $\bar{z}^{2} \bar{\lambda}(1-\exp \{-h \bar{\lambda}\}) \leq \bar{z}^{2} \bar{\lambda}$ and $E\left[\bar{Z}^{2}(t) \bar{\Lambda}(t)\right]$ is assumed finite (note that the distribution of $\bar{Z}^{2}(t) \bar{\Lambda}(t)$ depends on $\bar{h}$ and not $h$ ), it follows by dominated convergence that

$$
\lim _{h \downarrow 0} \frac{E\left[\bar{Z}^{2}(t) \bar{\Lambda}(t) h(1-\exp \{-h \bar{\Lambda}(t)\})\right]}{h}=E\left[\lim _{h \downarrow 0} \bar{Z}^{2}(t) \bar{\Lambda}(t)(1-\exp \{-h \bar{\Lambda}(t)\})\right]=0 .
$$

